\title{
Study on Control System in Opto-electronic Tracking Based on Sliding \\ Mode Variable Structure
}

\author{
Xiangru WANG ${ }^{a}$, Jianyong $Z_{H O N G}{ }^{b}$, Ranjun $W_{A N G}$ \\ Institute of Optics and Electronics, Chinese Academy of Sciences, Chengdu, 610209, China \\ amm9728@qq.com, bjyy@ioe.ac.cn, cwrj@ioe.ac.cn
}

\begin{abstract}
Keywords: opto-electrionic tracking, sliding mode variable structure, DC torque motor
Abstract. Aiming to solve the problem that DC torque motors is not steady to run at low speed in the control system of opto-electrionic tracking, sliding mode variable structure is applied to the low-speed servo system to improve stability of the system. At first the traditional design method of position, velocity and current loop control and Sliding Mode Variable Structure control are described. And their advantages and disadvantages are compared. Then the main factors affecting the performance of the low-speed opto-electronic servo system are analyzed. It's concluded that friction is the most important factor. And the concrete model of the friction is shown in mathematical model. Finally, the result of simulation by MATLAB indicates that Sliding Mode Variable Structure can achieve good control effect in the situation of DC torque motor running at low speed, which is superior to conventional design methods.
\end{abstract}

\section{Introduction}

In astronomical observation equipment weapons guidance systems and laser communication systems it requires precision photoelectric tracking device to make a precise point to the target. The control structure and design of algorithm in the tracking system is directly related to the performance of the system. It's a hot research about tracking system in the field of science in the whole world. Traditional design of current position, velocity loop control structure can often not achieve good effect in the applications of high speed and high precision. And in complex application it has poor anti-interference ability and poor stability in low speed applications. Therefore it's one of hot research of new control structures and algorithms to improve the opto-electronic tracking system.

In the early fifties of last century sliding mode variable structure (SMC) was put forward, it has the advantages such as not depending on accurate object model, simple method of design, high performance and well robustness. With fifty years of development, it is mainly used in the field of aerospace, robotics and servo motor control. The result of simulation by MATLAB shows that the synovial variable structure control in optical tracking system can achieve higher accuracy and better control robustness.

\section{The control structure of traditional opto-electronic tracking system}

The control structure of position, velocity and current loop is often used in opto-electronic tracking systems. As Fig. 1 shown, $C_{v}, C_{p}$ and $C_{i}$ represent the controllers of position loop, speed loop and current loop, $\mathrm{K}_{\mathrm{m}}$ is the DC motor torque coefficient, $\mathrm{C}_{\mathrm{e}}$ is the voltage feedback factor, $\mathrm{L}$ is the armature inductance, $\mathrm{R}$ is the armature resistance. 


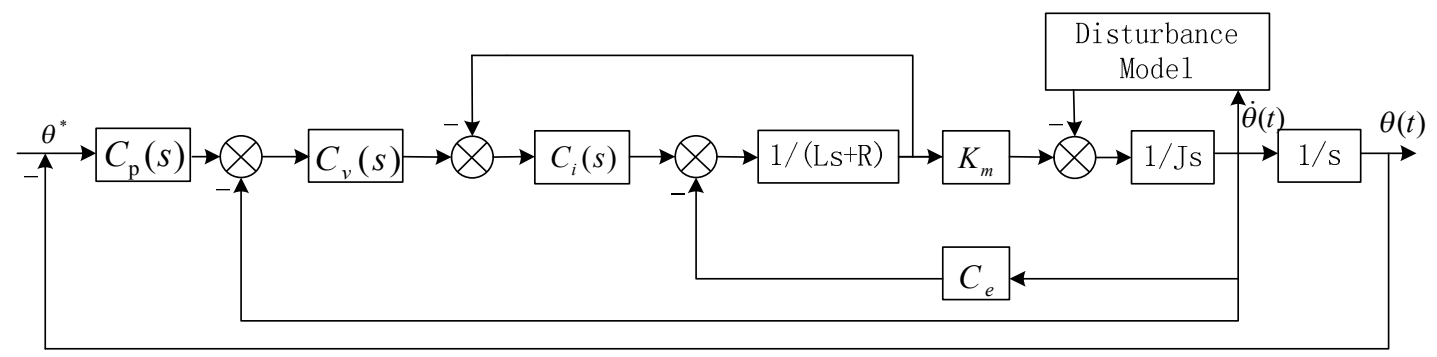

Fig.1 The control structure of position, velocity and current loop

The design of three-loop control structure in the tracking systems often start from the design of inner loop, and then according to the design specifications gradually the outer loops are designed. So in this article the design starts with the current loop, then the speed loop and position loop are designed in order.

Usually it takes the current loop controller $\mathrm{C}_{\mathrm{i}}$ for PI control as given below:

$$
C_{i}(s)=\frac{K_{i}\left(T_{m} s+1\right)}{s}
$$

where $\mathrm{K}_{\mathrm{i}}$ is the scaling factor, $\mathrm{T}_{\mathrm{m}}$ is the mechanical time constant.

As shown in Figure 1, it gets the transfer function of the current closed-loop and then simplifies to get:

$$
A(s)=\frac{1}{T_{i} s+1} \frac{K_{m}}{J_{S}}
$$

where $\mathrm{T}_{\mathrm{i}}$ is the function of electrical time constant and mechanical time constant.

As shown above, the current loop closed-loop transfer characteristics of the control object into a series of pure calculus and inertia. The electromagnetic time constant of the motor is reduced. It is possible to greatly improve the current loop bandwidth, further enhance the bandwidth of the system and weaken the effect on the system bandwidth caused by mechanical structural vibration frequency. And high current loop gain can weaken the influence of internal friction of the motor and improve motor torque stiffness.

Since the current loop is a typical type I model including an integrator, the speed controller $\mathrm{C}_{\mathrm{v}}(\mathrm{s})$ can be designed as a $\mathrm{P}$ regulator. Then the speed controller parameters $\mathrm{k}_{\mathrm{v}}$ can be designed based on the best second-order system.

Due to the cutoff frequency of the position loop is much smaller than the speed loop's, the speed closed loop can be reduced to a first-order inertia link. Position loop controller is given by:

$$
C_{p}(s)=\frac{K_{p}\left(T_{p} s+1\right)}{T_{q} s+1}
$$


The position open loop can be calculated by:

$$
W_{p}(s)=\frac{K_{w}\left(T_{p} s+1\right)}{s\left(T_{q} s+1\right)\left(T_{w}+1\right)}
$$

Finally, the position open loop function shows that it's a typical type I system, and the parameters of position controller can be commonly calculated based on the methods of frequency domain analysis in the engineering.

\section{The design of sliding mode variable structure}

Sliding mode variable structure is proposed by as an effective control method for nonlinear and uncertain system, which is suitable for application in complex electrical systems. It can't be effected by system parameter perturbation and external factors interfere. It has a smaller amount of calculation. It's easy to be designed than traditional design methods in practical applications. The design of sliding mode variable structure is shown as Fig.2.

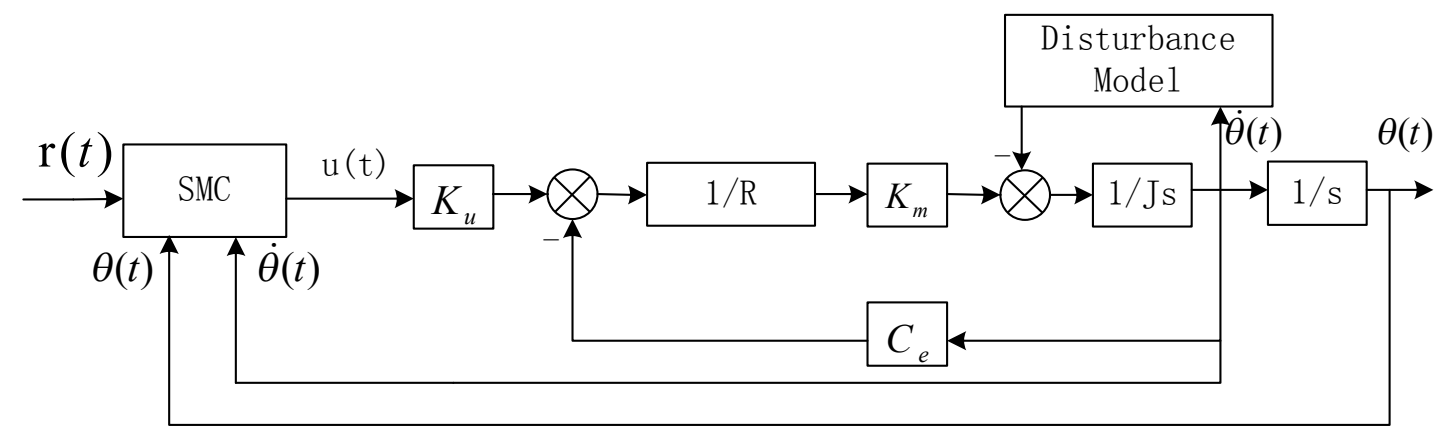

Fig.2 The control structure of sliding mode variable structure

Opto-electronic tracking system is essentially a DC torque motor servo system. While ignoring the armature inductance case, the relationship between its position and the voltage input is given by:

$$
\ddot{\theta}(t)=-\frac{K_{m} C_{e}}{J R} \dot{\theta}(t)+K_{u} \frac{K_{m}}{J R} u(t)-\frac{1}{J} F_{t}(t)
$$

Where $\theta(\mathrm{t})$ is the rotor position, $\mathrm{u}(\mathrm{t})$ is the input voltage.

Sliding mode variable structure based on exponential reaching law is often used in the tracking systems. This design method can not only shorten the time switching surface movement point approach, but also make the system movement speed small when it reaches the point of switching surface, therefore it reduces the impact of system chattering. Based on exponential reaching law synovial control design steps are shown as follows.

Firstly assume that $\mathrm{e}$ is the difference between given rotor position and the position feedback, the design of the switching function can be given by:

$$
s=c e+\dot{e}
$$


Secondly the exponential reaching law is depicted as:

$$
\dot{s}=-\varepsilon \operatorname{sgn}(s)-k s
$$

where $\varepsilon$ is greater than zero, $\mathrm{k}$ is greater than zero.

Thirdly the derivative of switching surface function is calculated as:

$$
\dot{s}=c \dot{e}+\ddot{e}
$$

Finally by equation (5), (7) and (8) we can get:

$$
u(t)=-\frac{J R}{K_{m} K_{u}}\left(c \dot{e}+\ddot{r}+\varepsilon \operatorname{sgn}(s)+k s+C_{e} \frac{K_{m}}{J R} \dot{\theta}(t)+\frac{1}{J} F_{f}(t)\right)
$$

where $r$ is the amount of the given location. We can get a faster response speed and higher control precision by adjusting reasonable parameter $\mathrm{c}$. We can weaken the affect of chattering robustness by adjust system parameters $\varepsilon$.

\section{Analysis on the factors of precision in the opto-electronic tracking system}

In the opto-electronic tracking systems three main factors generally have affect on accuracy of the system. The first one is the measurement noise sensor. It can achieve good results by adding circuit low-pass filter circuit in the measurement. The second is external vibration plat form, this can be solved by designing a mechanical structure isolated from external vibration. The third is the friction torque disturbance, which is one of the biggest factors affecting accuracy of the system. Opto-electronic tracking systems are commonly two-dimensional. In order to ensure the smooth when tilt and horizontal axes run at low speed, two shafts are usually installed tightly and friction between them is naturally large.

In friction analysis model stribeck model is common used, which exactly shows the relationship between static friction, coulomb friction, viscous friction and speed. Stribeck model can be depicted as follow:

$|\dot{\theta}|<\alpha$, the static friction is shown as:

$$
F_{f}(t)=\left\{\begin{array}{lr}
F_{m} & F(t)>F_{m} \\
F(t) & -F_{m}<F(t)<F_{m} \\
-F_{m} & F(t)<-F_{m}
\end{array}\right.
$$

$|\dot{\theta}|>\alpha$, dynamic friction is shown as: 


$$
F_{f}(t)=\left[F_{c}-\left(F_{m}-F_{c}\right) e^{\alpha_{1}|\dot{\theta}(t)|}\right] \operatorname{sgn}(\dot{\theta}(t))+k_{v} \dot{\theta}
$$

where is the speed of rotor, $F(t)$ is the driving force, $F_{m}$ is the maximum static friction, $F_{c}$ is Coulomb friction for, $\mathrm{k}_{\mathrm{v}}$ is viscous friction coefficient, both $\alpha_{1}$ and $\alpha$ are small positive numbers.

\section{Results and analysis of simulation}

A tracking system turntable's parameters are shown as: $R=7.77 \Omega, L=0.018 H, K_{m}=6 \mathrm{~N} \cdot \mathrm{m} / \mathrm{A}, \mathrm{C}_{\mathrm{e}}$ $=1.2 \mathrm{v} / \mathrm{A}, \mathrm{J}=0.6 \mathrm{Kg} / \mathrm{m} 2, \mathrm{~K}_{\mathrm{u}}=11 \mathrm{~V} / \mathrm{V}$. Friction parameters are shown as: $\mathrm{F}_{\mathrm{c}}=15 \mathrm{~N} \cdot \mathrm{m}, \mathrm{F}_{\mathrm{m}}=20$ $\mathrm{N} \cdot \mathrm{m}, \alpha_{1}=1, \mathrm{k}_{\mathrm{v}}=2 \mathrm{Nms} / \mathrm{rad}, \alpha=0.01$. According to the above theoretical model of the traditional control and sliding mode variable structure, simulation MATLAB simulink toolbox is used to analysis the two kinds of control models. Due to response to sinusoidal input signal can reflect the tracking performance of the actual system. It is possible to set both control models input sinusoidal signals. It is shown as: $\mathrm{r}(\mathrm{t})=0.1 \sin (2 \pi \mathrm{t})$. The simulation result is shown in Figure 3 and 4 .
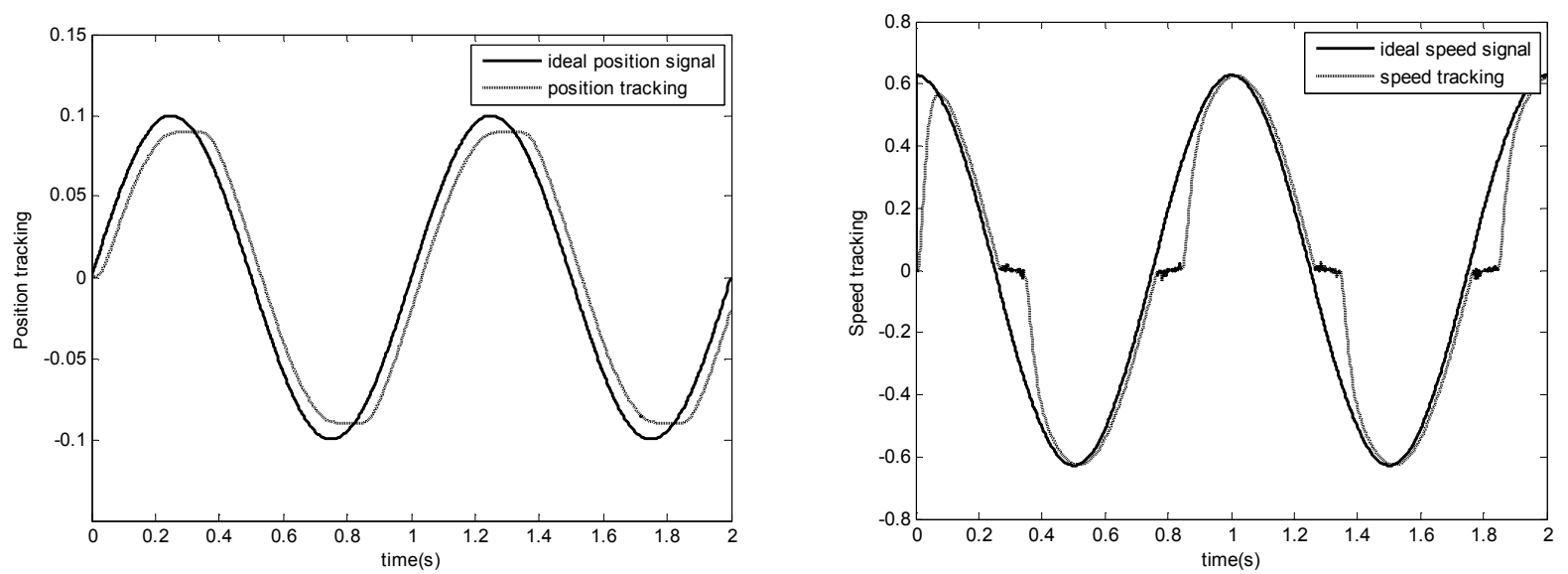

Fig. 3 position and velocity tracking curve of traditional three-loop control
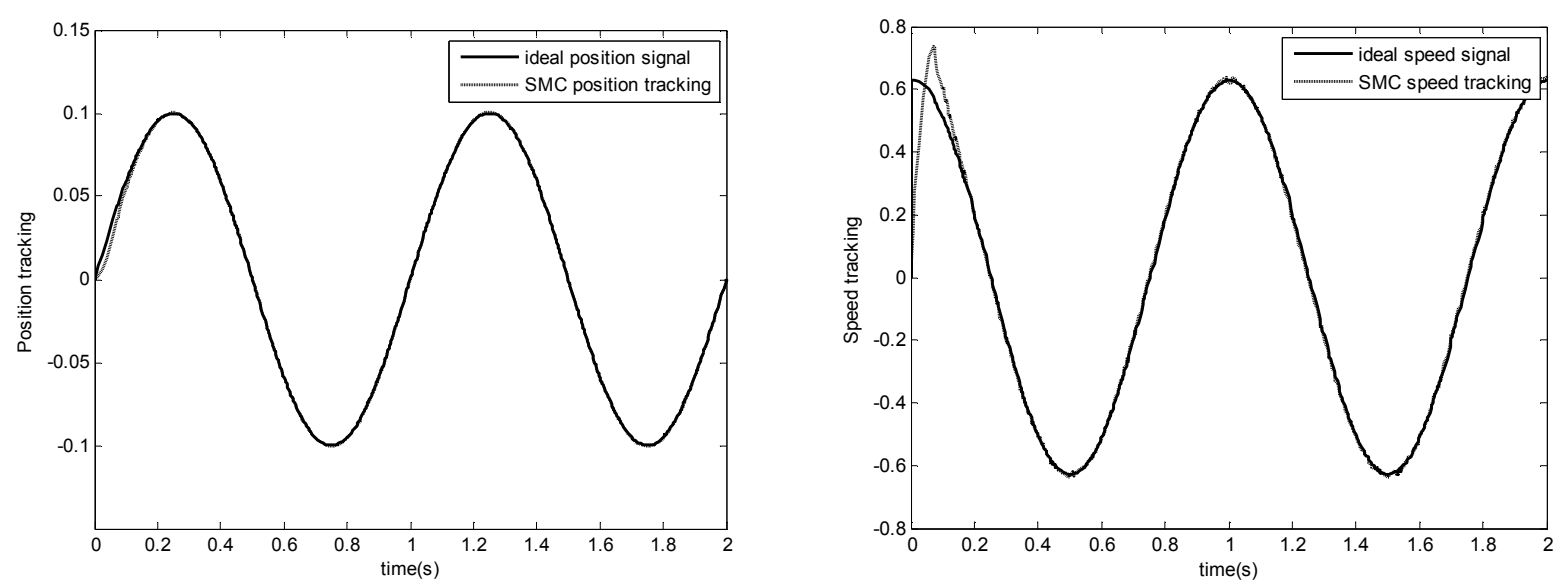

Fig.4 position and velocity tracking curve of sliding mode variable structure 
According to the position tracking curve in Fig.3 and Fig.4, the control method of sliding mode variable structure can get higher tracking accuracy than three-loop control's. And it virtually eliminates the hysteresis error and weakens effect on the accuracy of the position tracking caused by friction. The traditional three-loop control in the friction interference shows "flat top" phenomenon in position tracking curve near zero speed, and there is evident hysteresis in the tracking cure. Comparing both speed tracking curve we see small overshoot at the beginning in Fig 4 and input curve and track curve essentially coincident, while the traditional three-ring control speed track curve crossing the point of zero speed is not smooth due to the impact of the static friction.

\section{Conclusions}

According to the results of simulation applied with the actual control model the traditional three-loop controller can't meet the control requirement in the case of great friction interference, which affects the stability of the tracking system. And the sliding mode variable structure controller is simple to design. It can still achieve good control performance in the low-speed track under the influence of friction. In the future study sliding mode variable structure can be combined with other control methods so that it improves the tracking system.

\section{References}

[1] HUANG Yong-mei, ZHANG Tong, MA Jia-guang, FU Cheng-yu, Study on the control of a current loop in a high-accuracy tracking and control system, J. Opto-electric Engineering, 32(2005)16-19.

[2] YIN Ling-xiang, WANG Ai-xiang, The Applications of DC Torque Motors in Precision Tracking Radar, J. Electronics Engineering, 2001,55-57.

[3] PANG Xin-liang, ZHAO Wei-wei, FAN Da-peng, LI Chen-gang, LIU Yi-min, PENG Yan-hai, Application Research of DC Moment Motor on Airborne Opto-Electronic Servo System, J. Infrared Technique, 29(2007)573-578.

[4] LI Yong-jun, YANG Wen-shu, FAN Yong-kun, XIONG Ai, Controller Design and Simulation for Friction Moment Compensation on High-precision Turntable, J. Opto-electric Engineering, 35(2008)126-130.

[5] Du Bi-xiu, Mi Yang, Zhang Shumei, Application of variable structure control for high precision turntable, J. Foreign Electronic Measurement Technology, 33(2014)68-71.

[6] Ma Juan-li, Tang Yong-zhe, Zhi Xiao-bo, Study of Variable Structure Control with

Sliding-mode in Low-speed Friction Servo System, J. Journal of Projectiles, Rockets, Missiles and Guidance, 27(2008)77-80.

[7] Liu Jin-kun, MATLAB Simulation of Sliding Mode Control. Beijing, 2005. 